\title{
Neoadjuvant Chemotherapy Exerts Selection Pressure Towards Luminal Phenotype Breast Cancer
}

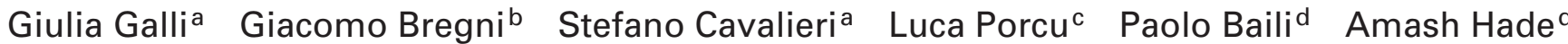 \\ Francesca Di Salvo $^{d}$ Milena Sant ${ }^{d}$ Roberto Agresti ${ }^{\mathrm{e}}$ Massimiliano Gennaro ${ }^{\mathrm{e}}$ Secondo Folli ${ }^{\mathrm{e}}$ \\ Maria C. De Santis ${ }^{f}$ Biagio Paolinig ${ }^{\text {Maria L. Carcangiug }}$ Filippo de Braud $^{\text {a }}$ Serena Di Cosimo $^{\text {h }}$ \\ a Department of Medical Oncology, Fondazione IRCCS Istituto Nazionale dei Tumori, Milano, Italy; \\ b Unit of Medical Oncology 1, IRCCS AOU San Martino-IST, Genova, Italy; \\ ${ }^{c}$ Computational Statistics Unit, Department of Oncology, IRCCS Istituto di Ricerche Farmacologiche Mario Negri, Milano, Italy; \\ ${ }^{d}$ Department of Preventive and Predictive Medicine, Fondazione IRCCS Istituto Nazionale dei Tumori, Milano, Italy; \\ e Department of General Surgery 3, Fondazione IRCCS Istituto Nazionale dei Tumori, Milano, Italy; \\ ${ }^{f}$ Department of Radiation Oncology 1, Fondazione IRCCS Istituto Nazionale dei Tumori, Milano, Italy; \\ g Department of Pathology 1, Fondazione IRCCS Istituto Nazionale dei Tumori, Milano, Italy; \\ h Dipartimento di Ricerca Applicata e Sviluppo Tecnologico (DRAST), Fondazione IRCCS Istituto Nazionale dei Tumori, Milano, Italy
}

\section{Keywords}

Breast cancer - Chemotherapy, neoadjuvant .

Endocrine therapy · Residual disease

\section{Summary}

Background: Breast cancer (BC) phenotype after neoadjuvant chemotherapy (NAC) has not been extensively described and few data exist on whether expression of the primary tumor hormone receptors, HER2 and Ki-67 changes as a result of chemotherapy. Materials and Methods: We analyzed specimens from all BC patients treated with anthracycline/taxane-based NAC at our Institution between January 2010 and March 2015 $(n=325)$. The expression of estrogen receptor (ER), progesterone receptor (PR), HER2 and $\mathrm{Ki}-67$ was determined in pre- and post-NAC specimens. McNemar's test was used to compare paired proportions. Results: Among patients with residual disease after NAC, basal phenotype was luminal $A$, luminal $B$, HER2 positive and triple negative in 44, 111, 74 and 27 cases, respectively. PR-positive tumors decreased from $68.0 \%$ in the initial biopsy sample to $61.7 \%$ in the surgical specimen $(p=0.024)$. A Ki-67 of $<20 \%$ increased from $23.6 \%$ to $45 \%$ ( $p<0.001$ ). ER expression changed from positive to negative in $5 \%$ and from negative to positive in $16.7 \%$ of cases. Overall, $30 \%$ of cases underwent subtype changes, $79 \%$ of them to-

Giulia Galli and Giacomo Bregni equally contributed to this work. wards luminal differentiation. Conclusions: The switch towards luminal phenotype suggests some kind of endocrine effect of NAC. Our findings raise renewed interest in combinatorial cytotoxic chemotherapy with concomitant or rather sequential endocrine therapy, either alone or with targeted agents.

(c) 2017 S. Karger GmbH, Freiburg

\section{Introduction}

Neoadjuvant chemotherapy (NAC) was originally introduced to reduce tumor size and enable surgery of locally advanced breast cancer (BC) [1]. Subsequent meta-analyses showed no differences in outcome between pre- and post-operative chemotherapy [2]. More recently, NAC has been considered as a unique opportunity to understand $\mathrm{BC}$ biology, thanks to the possibility of analyzing paired tumor samples before and after treatment [3]. The agents developed in this setting are similar to those validated in the adjuvant context, anthracyclines and taxanes being the most effective compounds [4]. Previous studies investigated the relation between Ki-67 drop and response to NAC. More recently, scientific interest was raised by the effects of NAC on hormone receptor (HR) and HER2 expression. Nevertheless, most previous works focused on small populations and various cytotoxic regimens. The objective of this study was to address $\mathrm{BC}$ phenotype changes in a homogeneous population of patients with localized or metastatic disease, mostly treated with anthracycline and taxane-based NAC within the same institution.

\section{KARGER}

() 2017 S. Karger GmbH, Freiburg 


\section{Materials and Methods}

We retrospectively collected information from the institutional database on all consecutive BC patients treated with NAC who underwent surgery at the Istituto Nazionale dei Tumori, Milan, Italy, between 1 January 2010 and 31 March 2015. For the current analysis we excluded all cases treated with chemotherapy in our Institution but undergoing surgery in other Institutions, as well as those without paired basal and surgical histological specimens and/or those treated with experimental therapy within a clinical trial. Data about patients' age, NAC regimen and duration, type and timing of surgery, clinical and pathological BC stage, and disease phenotype before and after NAC were extracted. All cases obtaining pathological complete response (pCR) were excluded from analysis. In absence of contraindications, all patients received anthracycline and taxane-based NAC. All HER2-positive cases were treated with trastuzumab, according to international guidelines. HR and HER2 status, Ki-67 index and disease stage were examined by Institutional pathologists. Diagnostic cut-offs for HR and HER2 positivity were defined according to American Society of Clinical Oncology-College of American Pathologists (ASCO-CAP) guidelines [5, 6]. Tumor subtype classification followed the St. Gallen/Vienna 2015 criteria [7]. All surgical specimens were completely sampled to detect residual disease. pCR was defined as the absence of invasive disease in both breast and lymph nodes. The primary objective of the study was to estimate percentage of changes in HR and HER2 status after NAC. Secondary objective was to compare percentage of cases with a specific tumor receptor status between pre- and post-NAC setting. 2-sided 95\% exact confidence intervals (CIs) were calculated to estimate percentage of patients changing BC subtype. The 2-sided exact McNemar's test was used to assess equality of basal and surgical paired percentages. Patients' and tumor characteristics, NAC regimens and disease response were summarized using descriptive statistics (median and range for continuous variables, absolute and percentage frequencies for categorical ones). Median follow-up was estimated using the reverse Kaplan-Meier method. Given the descriptive nature of the study, a quantitative hypothesis testing was applied instead that a formal one (e.g. no thresholds for statistical significance were defined). Statistical analysis was performed using SAS software, version 9.2 (SAS Institute, Cary, NC, USA).

\section{Results}

We identified 325 case with a median age of 51 years (range 23-85 years). Details about patients' and tumor characteristics are summarized in table 1 and table 2. 31 patients achieved pCR and were therefore excluded from analysis. Among the 294 patients with residual disease at surgery, paired tissue samples for determination estrogen receptor (ER) status at diagnostic biopsy and surgery were available in 274 cases (93.2\%). 11 of 220 (5.0\%, CI 2.5$8.8 \%$ ) patients showed a change from ER-positive to ER-negative disease. 9 of 54 (16.7\%, CI 7.9-29.3) patients converted from ERnegative to ER-positive disease. 34 of 183 (18.6\%, CI 13.2-25.0\%) cases with progesterone receptor (PR)-positive primary tumor had PR-negative residual disease at surgery. 17 of 86 (19.8\%, CI 12.029.8\%) cases with PR-negative disease showed PR-positivity at surgery. Among the 239 patients tested for HER2 in initial biopsy and surgical specimen, HER2 changed from positive to negative in 9 of 49 (18.4\%, CI 8.8-32.0\%) patients and from negative to positive in 7 of 190 (3.7\%, CI 1.5-7.4\%). Among the 229 cases with paired values of Ki-67, its expression decreased from $\geq 20 \%$ to $<20 \%$ in 63 of 175 (36.0\%, CI $28.9-43.6 \%)$ patients and increased from $<20 \%$ to $\geq 20 \%$ in 14 of 54 (25.9\%, CI $15.0-39.7 \%)$. The expression of PR and Ki-67 significantly differed between basal and surgical sam-
Table 1. Patients' characteristics

\begin{tabular}{|c|c|}
\hline \multicolumn{2}{|c|}{ Age at surgery, years } \\
\hline Median & 50.5 \\
\hline Range & $22.7-84.5$ \\
\hline \multicolumn{2}{|c|}{ ER status at diagnosis, $\mathrm{n}(\%)$} \\
\hline Positive & $239(74.0)$ \\
\hline Negative & $84(26.0)$ \\
\hline Missing & $2(0.6)$ \\
\hline \multicolumn{2}{|c|}{ PR status at diagnosis, $\mathrm{n}(\%)$} \\
\hline Positive & $198(62.3)$ \\
\hline Negative & $120(37.7)$ \\
\hline Missing & $7(2.2)$ \\
\hline \multicolumn{2}{|c|}{ HER2 status at diagnosis, $\mathrm{n}(\%)$} \\
\hline Positive & $90(28.6)$ \\
\hline Negative & $225(71.4)$ \\
\hline Missing & $10(3.1)$ \\
\hline \multicolumn{2}{|c|}{ Ki-67 status at diagnosis, $\mathrm{n}(\%)$} \\
\hline$\geq 20 \%$ & $228(79.7)$ \\
\hline$<20 \%$ & $58(20.3)$ \\
\hline Missing & $39(12.0)$ \\
\hline \multicolumn{2}{|c|}{ Tumor stage at diagnosis, $\mathrm{n}(\%)$} \\
\hline $\mathrm{X}$ & $1(0.3)$ \\
\hline $\mathrm{T} 1$ & $5(1.5)$ \\
\hline $\mathrm{T} 2$ & $184(56.6)$ \\
\hline $\mathrm{T} 3$ & $31(9.5)$ \\
\hline $\mathrm{T} 4$ & $104(32.0)$ \\
\hline \multicolumn{2}{|c|}{ Nodal stage at diagnosis, n (\%) } \\
\hline N0 & $95(29.2)$ \\
\hline N1 & $199(61.2)$ \\
\hline N2 & $22(6.8)$ \\
\hline \multicolumn{2}{|c|}{ Metastasis at diagnosis, n (\%) } \\
\hline M0 & $285(87.7)$ \\
\hline M1 & $40(12.3)$ \\
\hline
\end{tabular}

Table 2. Treatment characteristics

\begin{tabular}{lc} 
Cytotoxic regimen, $\mathrm{n}(\%)$ & \\
Anthracycline and taxane & $292(91.0)$ \\
Anthracycline alone & $9(2.8)$ \\
Taxane alone & $12(3.7)$ \\
Other & $8(2.5)$ \\
Missing & $4(1.2)$ \\
Cycles administered & \\
Median, $\mathrm{n}$ & 6 \\
Range, $\mathrm{n}$ & $2-18$ \\
Missing, n (\%) & $9(2.8)$ \\
Type of surgery, $\mathrm{n}(\%)$ & \\
Modified radical mastectomy & $221(68.0)$ \\
Conservative surgery & $104(32.0)$ \\
\hline
\end{tabular}

ples. Indeed, PR positivity was reported in $68.0 \%$ (CI 62.1-73.6\%) of diagnostic biopsy samples and in $61.7 \%$ (CI 55.6-67.5\%) of surgical specimens (McNemar's test $\mathrm{p}=0.024$ ). Ki-67 was $\geq 20 \%$ in $76.4 \%$ (CI 70.4-81.8\%) of pre-treatment biopsies and in $55.0 \%$ (CI 48.3-61.6\%) of residual diseases (McNemar's test $\mathrm{p}<0.001$ ). Overall, 62 of $206(30.1 \%)$ cases presented a phenotype modification from basal biopsy to surgical specimen. In particular, of these 206 
Table 3. Immunohistochemistry in initial biopsy and surgical specimens

\begin{tabular}{|c|c|c|c|c|c|}
\hline \multirow[t]{2}{*}{ Initial biopsy } & \multicolumn{2}{|c|}{ Final surgery, n } & \multicolumn{2}{|c|}{ Prevalence, \% (95\%CI) } & \multirow[t]{2}{*}{$\mathrm{p}$-value } \\
\hline & $\begin{array}{l}\text { positive } \\
(\geq 20 \%)\end{array}$ & $\begin{array}{l}\text { negative } \\
(<20 \%)\end{array}$ & initial biopsy & final surgery & \\
\hline \multicolumn{6}{|l|}{ ER status } \\
\hline Positive & 209 & 11 & $80.3(75.1-84.8)$ & $79.6(74.3-84.2)$ & 0.824 \\
\hline Negative & 9 & 45 & $19.7(15.2-24.9)$ & $20.4(15.6-25.7)$ & \\
\hline \multicolumn{6}{|l|}{ PR status } \\
\hline Positive & 149 & 34 & $68.0(62.1-73.6)$ & $61.7(55.6-67.5)$ & 0.024 \\
\hline Negative & 17 & 69 & $32.0(26.4-37.9)$ & $38.3(32.5-44.4)$ & \\
\hline \multicolumn{6}{|l|}{ HER2 status } \\
\hline Positive & 40 & 9 & $20.5(15.6-26.2)$ & $19.7(14.8-25.3)$ & 0.804 \\
\hline Negative & 7 & 183 & $79.5(73.8-84.4)$ & $80.3(74.7-85.3)$ & \\
\hline \multicolumn{6}{|l|}{ Ki-67 } \\
\hline$\geq 20 \%$ & 112 & 63 & $76.4(70.4-81.8)$ & $55.0(48.3-61.6)$ & $<0.001$ \\
\hline$<20 \%$ & 14 & 40 & $23.6(18.2-29.6)$ & $45.0(38.4-51.7)$ & \\
\hline
\end{tabular}

\begin{tabular}{|c|c|c|c|c|c|}
\hline \multirow[t]{2}{*}{ Basal histotype, $n$} & & \multicolumn{4}{|c|}{ Post-treatment histotype, n (\%) } \\
\hline & & unchanged & changed & & missing data \\
\hline \multirow[t]{2}{*}{ Triple negative } & 27 & $23(95.8)$ & luminal B & $1(4.2)$ & $3(11.1)$ \\
\hline & & & triple negative & $2(4.2)$ & $26(35.1)$ \\
\hline \multirow[t]{2}{*}{ HER2 positive } & 74 & $40(83.3)$ & luminal A & $4(8.3)$ & \\
\hline & & & luminal $B^{*}$ & $2(4.2)$ & \\
\hline Luminal A & 44 & $31(77.5)$ & luminal B & $9(22.5)$ & $4(9.1)$ \\
\hline \multirow[t]{3}{*}{ Luminal B } & 111 & $50(53.2)$ & triple negative & $5(5.3)$ & $17(15.3)$ \\
\hline & & & HER2 positive & $6(6.4)$ & \\
\hline & & & luminal A & $33(35.1)$ & \\
\hline
\end{tabular}

${ }^{*} 2 / 2$ cases changed from HER2-positive to luminal B HER2-positive (triple positive) breast cancer. patients, 24 were triple-negative (11.7\%) at baseline and $30(14.6 \%)$ after NAC (McNemar's test $\mathrm{p}=0.070)$; 48 were HER2 positive $(23.3 \%)$ at baseline and $46(22.3 \%)$ after NAC (McNemar's test $\mathrm{p}=0.790)$; 40 were luminal A (19.4\%) at baseline and $68(33.0 \%)$ after NAC (McNemar's test $\mathrm{p}<0.0001)$; and 94 luminal B $(45.6 \%)$ at baseline and $62(30.1 \%)$ after NAC (McNemar's test $\mathrm{p}<0.0001)$. Most cases showing any kind of phenotype change presented locally advanced or metastatic disease as, of the 62, 30 (48.4\%) were pT3-pT4, 49 (79.0\%) were pN+ and 11 (17.7\%) presented synchronous distant metastases. Tumor marker and phenotype changes in paired samples are reported in table 3 and table 4 .

\section{Discussion}

Change in HR and HER2 status after NAC is a well-known phenomenon in BC, occurring in $10-30 \%$ of cases $[8,9]$. Although the older results might be affected by pre-analytical and analytical pitfalls $[10,11]$, a real biological heterogeneity may be implied. It was hypothesized that paired samples from a single lesion may differ, even in absence of therapeutic interventions, as a consequence of malignant subpopulations coexistence. Some studies supported this theory, identifying no differences in phenotype change rates between paired samples from treatment-naïve and chemo-treated patients $[12,13]$. An alternative hypothesis supports a drug-induced selection pressure towards malignant cells. Cytotoxic, endocrine and biological agents may specifically target and kill sensitive $\mathrm{BC}$ subpopulations, leaving behind the resistant ones. This hypothesis was sustained by some studies, showing higher frequency of $\mathrm{BC}$ phenotype change in NAC-treated patients than in naïve controls $[14,15]$. The present work shows that most cases undergoing phenotype changes presented with advanced stage ( $\mathrm{pT} 3-4, \mathrm{pN}+$, $\mathrm{cM}+$ ). This observation is in favor of the hypothesis that treatment might possibly affect part of a rather heterogeneous disease, leading to the emergence of an unresponsive residue. On the other hand, a considerable amount of data suggests that Ki-67 drop after NAC may be an independent positive prognostic factor in patients not achieving pCR $[16,17]$. In the present study, we observed a significant decrease in PR and Ki-67 expression after NAC. This finding seems to support a prognostic, rather than an incidental role of BC phenotype change during NAC. Previous works postulated that cytotoxic agents may play an endocrine role, suppressing ovarian and adrenal function with consequent alteration of $\mathrm{BC}$ biological profile [18]. Indeed, it was shown that pre-menopausal 
women with HR-positive tumors attaining amenorrhea after NAC had better outcome than menstruating women [19]. Post-menopausal patients may also achieve benefit, due to adrenal endocrine suppression induced by cytotoxic agents [20]. If confirmed, these hypotheses may entail important clinical implications, as PR decrease may be viewed as a marker of reduced endocrine stimulation in BC. Another finding of this study concerns the tendency towards luminal differentiation observed after NAC. Even though our data do not allow prognostic or pathogenetic conclusions to be drawn, this previously unreported finding is in line with the hypothesis of an endocrine effect of chemotherapy. This observation potentially raises new interest in the development of sequential cytotoxic and hormonal NAC approaches, or combinations with innovative drugs such as cyclin kinase inhibitors, to target biological modifications of tumor phenotype induced by standard NAC. A plethora of gene expression profiles have been generated during the last decades to discover, develop and validate prognostic and predictive gene signatures. Some of these signatures are commercially available to define the residual risk in ER-positive and HER2negative tumors after receiving adjuvant endocrine treatment (Mammaprint, Oncotype DX, Breast Cancer Index, PAM50, EndoPredict) [21]. Unfortunately, none of these signatures were aimed at specifically defining the residual risk after standard NAC. Our findings obtained using the St. Gallen criteria for BC classification suggest that gene expression profiling of residual disease could help to further characterize the modifications induced by anthracycline/taxane NAC, with the final goal of defining patients at high recurrence risk despite standard treatment, who could be ideal candidates for trials with investigational drugs in early breast cancer. A significant value of our study consists in the focus on a homogeneous population, treated over a limited period of time. Furthermore, the cytotoxic and biological agents administered were in agreement with recent guidelines on NAC. However, some limitations of this work have to be underlined. First of all, it represents a retrospective case series based on a limited number of patients. Secondly, immature follow-up does not allow outcome conclusions to be drawn. Finally, this study was conceived as an explorative analysis without pre-defined postulates, with a speculative and hypothesis-generating aim.

In conclusion, the reported data suggest that NAC may induce distinct phenotypical changes in BC. We believe this observation deserves further evaluation as it may entail crucial implications in the development of new neoadjuvant therapeutic strategies.

\section{Disclosure Statement}

The Authors have no relevant conflicts of interest to disclose.

\section{References}

1 Kaufmann M, von Minckwitz G, Smith R, et al.: International expert panel on the use of primary (preoperative) systemic treatment of operable breast cancer: review and recommendations. J Clin Oncol 2003;21: 2600-2608.

2 Gianni L, Baselga J, Eiermann W, et al.: Phase III trial evaluating the addition of paclitaxel to doxorubicin followed by cyclophosphamide, methotrexate, and fluorouracil, as adjuvant or primary systemic therapy: European Cooperative Trial in Operable Breast Cancer. J Clin Oncol 2005;27:2474-2481.

3 Schott AF, Hayes DF: Defining the benefits of neoadjuvant chemotherapy for breast cancer. J Clin Oncol 2012;20:1747-1749.

4 Haddad TC, Goetz MP: Landscape of neoadjuvant therapy for breast cancer. Ann Surg Oncol 2015;22: 1408-1415.

5 Hammond ME, Hayes DF, Wolff AC: American Society of Clinical Oncology/College of American Pathologists guideline recommendations for immunohistochemical testing of estrogen and progesterone receptors in breast cancer. J Clin Oncol 2010;28:2784-2797.

6 Wolff AC, Hammond ME, Hicks DG, et al.: Recommendations for human epidermal growth factor receptor 2 testing in breast cancer: American Society of Clinical Oncology/College of American Pathologists clinical practice guideline update. J Clin Oncol 2013; 25:118-145.
7 Vasconcelos I, Hussainzada A, Berger S, et al.: The St. Gallen surrogate classification for breast cancer subtypes successfully predicts tumor presenting features, nodal involvement, recurrence pattern and disease free survival. Breast 2016;31:3997-4013.

8 Parinyanitikul N, Lei X, Chavez-MacGregor M, et al.: Receptor status change from primary to residual breast cancer after neoadjuvant chemotherapy and analysis of survival outcomes. Clin Breast Cancer 2015;15:153-160.

9 Tacca O, Penault-Llorca F, Abrial C, et al.: Changes in and prognostic value of hormone receptor status in a series of operable breast cancer patients treated with neoadjuvant chemotherapy. Oncologist 2007;12:636643.

10 Morris M, Edwards J, Gelder F: Hormonal receptors in locally advanced breast cancer: Change with response to neoadjuvant chemotherapy? J Surg Oncol 1991;46: 156-158.

11 Lo SS, Wang HC, Shyr YM, Lui WY: Can hormonal receptor status of primary breast cancer be altered by neoadjuvant chemotherapy? J Surg Oncol 1994;57:9496.

12 Lee SH, Chung MA, Quddus MR, et al.: The effect of neoadjuvant chemotherapy on estrogen and progesterone receptor expression and hormone receptor status in breast cancer. Am J Surg 2003;186:348-350.

13 Zhou X, Zhang J, Yun H, et al.: Alterations of biomarker profiles after neoadjuvant chemotherapy in breast cancer: Tumor heterogeneity should be taken into consideration. Oncotarget 2015;6:36894-36902.
14 Hirata T, Shimizu C, Yonemori K, et al.: Change in the hormone receptor status following administration of neoadjuvant chemotherapy and its impact on the long term outcome in patients with primary breast cancer. Br J Cancer 2009;101:1529-1536.

15 Yang YF, Liao YY, Li LQ, et al.: Changes in ER, PgR and HER2 receptors status after neoadjuvant chemotherapy in breast cancer. Pathol Res Pract 2013;209:797-802.

16 von Minckwitz G, Schmitt WD, Loibl S, et al.: Ki67 measured after neoadjuvant chemotherapy for primary breast cancer. Clin Cancer Res 2013;19:4521-4531.

17 Montagna E, Bagnardi V, Viale G, et al.: Changes in $\mathrm{PgR}$ and $\mathrm{Ki}-67$ in residual tumour and outcome of breast cancer patients treated with neoadjuvant chemotherapy. Ann Oncol 2015;26:307-313.

18 Jin X, Jiang YZ, Chen S, et al.: Prognostic value of receptor conversion after neoadjuvant chemotherapy in breast cancer patients: A prospective observational study. Oncotarget 2015;6:9600-9611.

19 International Breast Cancer Study Group, Colleoni M Gelber S, et al.: Tamoxifen after adjuvant chemotherapy for premenopausal women with lymph node-positive breast cancer: International Breast Cancer Study Group Trial 13-93. J Clin Oncol 2006;24:1332-1341.

20 Rose DP, Davis TE: Effects of adjuvant chemohormonal therapy on the ovarian and adrenal function of breast cancer patients. Cancer Res 1980;40:4043-4047.

21 Kwa M, Makris A, Esteva FJ: Clinical utility of geneexpression signatures in early stage breast cancer. Nat Rev Clin Oncol 2017;14:595-610. 